\title{
Development of a siRNA and shRNA screening system based on a kinase fusion protein
}

\author{
SOFIE PIERAETS, ${ }^{1,2}$ LUK COX, ${ }^{1,2}$ OLGA GIELEN, ${ }^{1,2}$ and JAN COOLS ${ }^{1,2,3}$ \\ ${ }^{1}$ Center for the Biology of Disease, VIB, B-3000 Leuven, Belgium \\ ${ }^{2}$ Center for Human Genetics, KU Leuven, B-3000 Leuven, Belgium
}

\begin{abstract}
RNA interference (RNAi) is one of the processes in the cell that regulates mRNA expression levels. RNAi can be exploited to experimentally knockdown the expression of one or more genes in cell lines or even in cells in vivo and also became an interesting tool to develop new therapeutic approaches. One of the major challenges of using RNAi is selecting effective shRNAs or siRNAs that sufficiently down-regulate the expression of the target gene. Here, we describe a system to select functional shRNAs or siRNAs that makes use of the leukemia cell line Ba/F3 that is dependent on the expression of a mutant form of the PDGFR $\alpha$ kinase for its proliferation and survival. The basis of this system is the generation of an expression construct, where part of the open reading frame of the gene of interest is linked to the mutant PDGFR . Thus, shRNAs or siRNAs that effectively target the gene of interest also result in a reduction of the expression of the mutant PDGFR $\alpha$ protein, which can be detected by a reduction of the proliferation of the cells. We demonstrate that this validation system can be used for the selection of effective siRNAs as well as shRNAs. Unlike other systems, the system described here is not dependent on obtaining high-transduction efficiencies, and nonspecific effects of the siRNAs or shRNAs can be detected by comparing the effects in the presence or absence of the growth factor interleukin-3.
\end{abstract}

Keywords: RNA interference; kinase; cell-based assay; proliferation; screening system

\section{INTRODUCTION}

RNA interference (RNAi) is the process of gene silencing that occurs in a sequence-specific manner at the level of the mRNA (Siomi and Siomi 2009). The basic process of RNAi can be divided into three steps. Initially, the ribonuclease III enzyme Dicer cleaves a long double-stranded RNA that is expressed or introduced into the cell into small RNAs (2030 nucleotides). Secondly, one strand of the small RNA, the guided strand, is loaded onto the RNA-induced silencing complex (RISC), while the other, the passenger strand, is degraded. Finally, the guided strand binds the target mRNA, which results in the degradation of the mRNA. Human cells express hundreds of noncoding RNAs that are processed in the cell to short RNAs that enter the RNAi pathway and are important for gene-expression regulation (Winter et al. 2009; Krol et al. 2010; Sashital and Doudna 2010). Synthesized double-stranded short interfering RNAs (siRNAs) introduced into the cell or short hairpin RNAs (shRNAs)

\footnotetext{
${ }^{3}$ Corresponding author.

E-mail jan.cools@cme.vib-kuleuven.be.

Article published online ahead of print. Article and publication date are at http://www.rnajournal.org/cgi/doi/10.1261/rna.030015.111.
}

or miRNA-based shRNAs (shRNAmirs) expressed in the cell use the cellular mechanism of RNAi to interfere with the expression of a specific gene, and in this way create a cell that lacks sufficient expression levels of that gene (Rao et al. 2009; Siomi and Siomi 2009; Krol et al. 2010; Sashital and Doudna 2010).

In the lab RNAi is used as a research tool in the siRNA and shRNA/shRNAmir mode. Such siRNAs are $\sim 21-25$ nucleotides long and are introduced artificially by means of transfection or electroporation. The shRNAs and shRNAmirs are typically incorporated in expression vectors such as plasmids or retroviral/lentiviral vectors, through which the shRNAs or shRNAmirs are stably integrated in the host genome. Those synthesized small RNA molecules use the endogenous RNAi pathway in eukaryotic cells for the degradation of their target mRNA (Rao et al. 2009; Siomi and Siomi 2009; Krol et al. 2010; Sashital and Doudna 2010; Pecot et al. 2011).

Despite the general use of RNAi in research, there are still a number of limitations and difficulties associated with this technology. First of all, delivery of siRNAs to cells in vitro, and even more in vivo, remains a bottleneck for the widespread use of RNAi. Second, despite the specificity due to the sequence of the siRNAs or shRNAs, off-target effects 
are present and may cause unwanted and difficult to control side effects. Third, the selection of siRNA or shRNA sequences that are effective in down-regulating the expression of the target genes remain difficult (Elbashir et al. 2001; Holen et al. 2002; Kapadia et al. 2003; Reynolds et al. 2004; Pecot et al. 2011). Although design algorithms have improved in the last years, there are still difficulties in identifying effective and specific siRNAs and shRNAs. Therefore, it is still required to experimentally validate every siRNA or shRNA that is used, and for a specific gene 10 or more sequences may need to be tested to identify one RNAi molecule that is causing sufficient knockdown (Pei and Tuschl 2006; Krueger et al. 2007; Wang et al. 2009).

To assist in the identification of functional siRNAs or shRNAs, a number of experimental validation systems have been developed. Those validation systems consist of a reporter assay where a target gene is fused to a fluorescent or enzymatic reporter. In these systems, expression of functional shRNAs or siRNAs results in a decrease of the reporter signal, and thus loss of fluorescent or enzymatic activity is a measure for the knockdown efficiency of the siRNAs or shRNAs (Kumar et al. 2003; Huesken et al. 2005; Zhao et al. 2005; Hung et al. 2006; Kasim et al. 2006; Malik et al. 2006; Luo et al. 2007; Fellmann et al. 2011).

In this work we describe the development of a protein-kinase fusion system in $\mathrm{Ba} / \mathrm{F} 3$ cells that is based on a proliferation read-out to test the knockdown efficiency of RNAi molecules.

\section{RESULTS}

\section{Development of the $\mathrm{Ba} / \mathrm{F} 3$ validation system}

In order to evaluate the silencing capacity of selected shRNAs or siRNAs in a medium to high throughput system, we developed a cell-based system in which shRNA/siRNA molecules that efficiently knockdown the expression of a given gene would inhibit cell proliferation. In this way, the silencing effect can be read from the effect on cell proliferation, as is also done in cell-based drug screens (Fig. 1). For this, we used the interleukin-3 (IL-3)-dependent pro B cell line $(\mathrm{Ba} / \mathrm{F} 3)$. These $\mathrm{Ba} / \mathrm{F} 3$ cells are dependent on IL-3 for their proliferation and survival, but can also become dependent on oncogenic tyrosine kinases such as activated ABL, FLT3, PDGFR $\alpha$, and many other kinases (Lierman et al. 2007; Warmuth et al. 2007). For our system, we used a truncated form of PDGFR $\alpha$ (named "tPDGFR $\alpha$ "), known to be constitutively activated and to stimulate the proliferation and survival of $\mathrm{Ba} / \mathrm{F} 3$ cells in the absence of IL-3 (Cools et al. 2003a,b; Stover et al. 2006; Warmuth et al. 2007).

Based on our previous experiences with the $\mathrm{Ba} / \mathrm{F} 3$ cell system, we reasoned that this cell system would be a good screening system to select efficient siRNA or shRNA sequences. Ba/F3 cells transformed by tPDGFR $\alpha$ have a short doubling time and are absolutely dependent on the activated kinase for their proliferation and survival. A siRNA targeting
tPDGFR $\alpha$ can be used as a positive control in the screens, and IL-3 can be added to the cells to make them independent of tPDGFR $\alpha$ in order to distinguish between specific and toxic off-target effects of the siRNAs or shRNAs. Finally, the tPDGFR $\alpha$ can be fused to additional sequences without affecting its function (Stover et al. 2006). In this way, the gene of interest (the gene that one wants to target) can be put on the same transcript as $t P D G F R \alpha$ (Figs. 1, 2A).

Three different variants of the vector were generated: variant 1 with the target gene upstream of $t P D G F R \alpha$, generating a fusion protein between the coding sequence of the target gene and $t P D G F R \alpha$; variant 2 with the target gene downstream from $t P D G F R \alpha$, with a fusion between $t P D G F R \alpha$ and the coding sequence of the target gene; and variant 3 with the target gene downstream from $t P D G F R \alpha$ on the same RNA, but without generating a fusion protein (Fig. $2 \mathrm{~A})$. Thus, the first two variants result in the expression of a fusion protein of tPDGFR $\alpha$ with the protein of interest (or a part of the protein of interest), while in the third version the target gene is present as the $3^{\prime}$ untranslated region (UTR) of tPDGFR $\alpha$ (Figs. 1, 2A).

\section{Selection of shRNAs targeting $p 16$ and Pten using the $\mathrm{Ba} / \mathrm{F} 3$ system}

In order to test the $\mathrm{Ba} / \mathrm{F} 3$ system, we designed shRNAs targeting mouse Pten and mouse Cdkn2a (p16), and tested whether the $\mathrm{Ba} / \mathrm{F} 3$ system could be used to select the most potent shRNA. We generated tPDGFR $\alpha$ constructs containing the coding sequences of Cdkn2a (p16) and Pten (Fig. 2A). These constructs were retrovirally transduced in $\mathrm{Ba} / \mathrm{F} 3$ cells. As such, six different $\mathrm{Ba} / \mathrm{F} 3$ cell lines were generated to test the system.

All six newly generated $\mathrm{Ba} / \mathrm{F} 3$ cell lines could grow in the absence of IL-3 at the same proliferation rate as the control cells expressing only the truncated form of PDGFR $\alpha$ (Fig. 2B). Western blot analysis revealed autophosphorylation of all tPDGFR $\alpha$ fusion proteins in these different cell lines, indicating that all cell lines expressed activated PDGFR $\alpha$ as expected (Fig. 2C). In addition, the growth of all cell lines was inhibited by the PDGFR $\alpha$ kinase inhibitor imatinib, further confirming that the cell lines were all strictly dependent on the expression of the activated PDGFR $\alpha$ (Fig. 2D). In another experiment we showed that after electroporation with an effective siRNA against $t P D G F R \alpha$ those cell lines were again dependent on IL-3 for their cell growth (Fig. 2E).

To test the potency of the different retroviral shRNA vectors, we transduced the variant $\mathrm{Ba} / \mathrm{F} 3$ cells with these retroviral vectors and followed the effect on the proliferation of the transduced cells. Since the shRNA vectors also express green fluorescent protein (GFP), we could identify shRNAexpressing cells using flow cytometry, which allowed us to trace the fate of the transduced cells over time. If the percentage GFP positive cells decreased over time, this was indicative of effective knockdown of the target RNA, since 


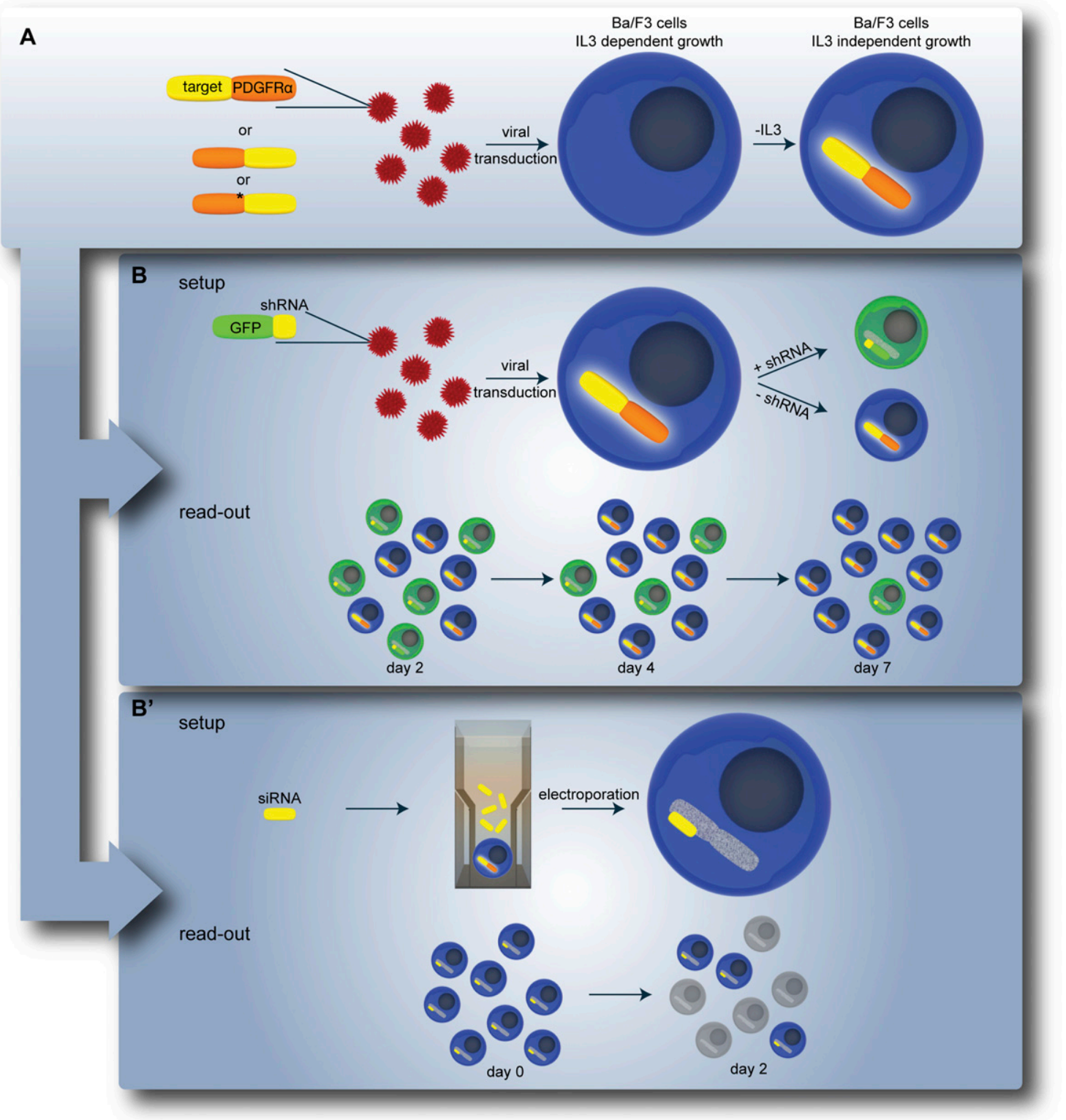

FIGURE 1. Schematic overview of the use of the validation system for selecting functional shRNAs or siRNAs. (A) Ba/F3 cells expressing tPDGFR $\alpha$ or a fusion of tPDGFR $\alpha$ with a gene of interest can grow in the absence of the growth factor IL-3. These Ba/F3 cells are completely dependent on the expression of the mRNA containing the tPDGFR $\alpha$ open reading frame. The gene of interest is placed on the same mRNA, either upstream of or downstream from the $t P D G F R \alpha$. The asterisk indicates the presence of a stop codon. (B) Transduction of the Ba/F3 cells with shRNAs (coexpressing GFP) that effectively down-regulate the gene of interest, and thus prevent expression of the tPDGFR $\alpha$, will result in a decrease of the number of GFP positive cells in the culture. $\left(B^{\prime}\right)$ Electroporation of Ba/F3 cells with siRNAs that effectively down-regulate the gene of interest, and thus prevent expression of the tPDGFR $\alpha$, will inhibit the proliferation of the cells.

the target RNA is on the same RNA as $t P D G F R \alpha$, which is required for the proliferation of the cells (Fig. 1).

In a first set of experiments we used the $\mathrm{Ba} / \mathrm{F} 3$ cell lines with the open reading frames of $p 16$ or Pten positioned upstream of $t P D G F R \alpha$ and could demonstrate a clear distinction between effective and noneffective shRNAs. In the cells expressing p16-tPDGFR $\alpha$, the strongest decrease of GFP positive cells was obtained with shRNA-p16-1. ShRNA-p16-3 was less efficient, and no effect was observed for shRNA- p16-2 at the endpoint of the experiment $11 \mathrm{~d}$ after transduction. No significant change in the GFP positive population was observed in the control cells that expressed tPDGFR $\alpha$ or the cells containing the Pten-tPDGFR $\alpha$ fusion (Fig. 3A).

When targeting the cells expressing Pten-tPDGFR $\alpha$, all three shRNAs targeting Pten were found to be effective, with the strongest effect observed for shRNA-Pten-4. To rule out that the effects observed on the proliferation of the $\mathrm{Ba} / \mathrm{F} 3$ cells was due to more general toxic effects, we also tested 
A

variant 1 myc 1 tran

B

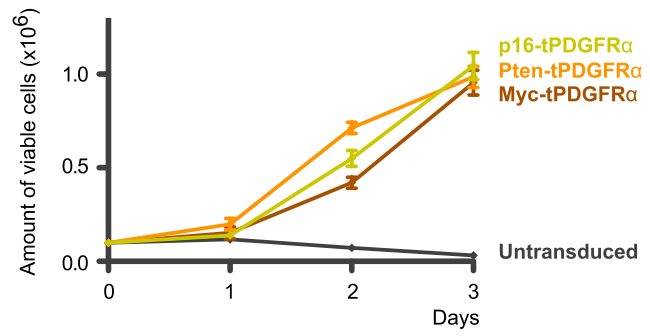

D

C

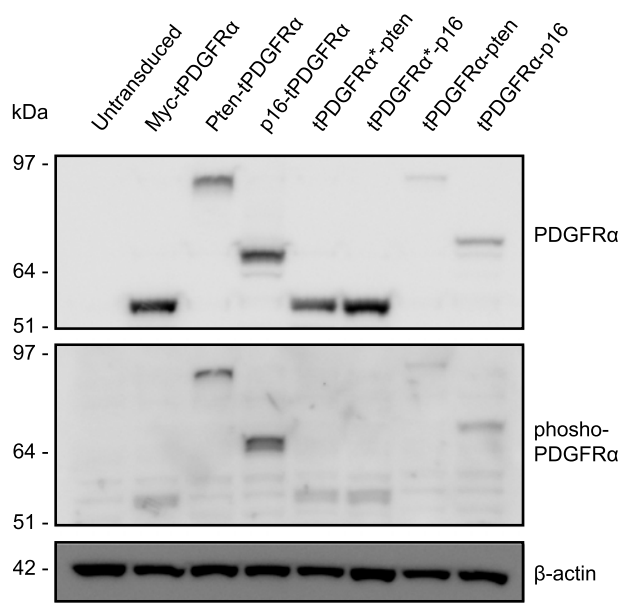

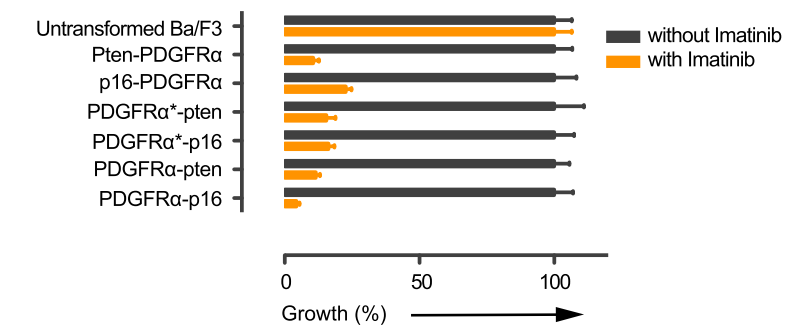

$\mathbf{E}$

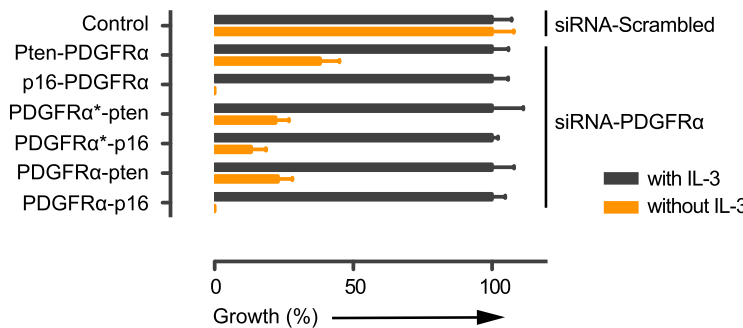

FIGURE 2. Expression of all constructs containing the tPDGFR $\alpha$ in Ba/F3 cells results in IL-3 independent growth. (A) Schematic representation of the different constructs: myc-Pten-tPDGFR $\alpha$, myc-p16-tPDGFR $\alpha$, myc-tPDGFR $\alpha$-Pten and myc-tPDGFR $\alpha$-p16, myc-tPDGFR $\alpha^{*}$-Pten and myc-tPDGFR $\alpha^{\star}$-p16. The target genes Pten and $p 16$ were cloned upstream of the $t P D G F R \alpha$, and downstream with a stop codon $\left({ }^{\star}\right)$ in between. (B) $\mathrm{Ba} / \mathrm{F} 3$ cells transduced with the tPDGFR $\alpha$ constructs grow in the absence of IL-3. Untransduced Ba/F3 cell do not survive without IL-3. Mean growth \pm SEM was recorded in triplicate for $3 \mathrm{~d}$. (C) All transformed $\mathrm{Ba} / \mathrm{F} 3$ cells showed expression (top) and phosphorylation (middle) of the tPDGFR $\alpha$. Expression of $\beta$-actin (bottom) is used as control for the loading. (D) Compared with the untransformed cells, growth of the transformed $\mathrm{Ba} / \mathrm{F} 3$ cells was reduced when treated with $100 \mathrm{nM}$ PDGFR $\alpha$ kinase inhibitor imatinib. Mean growth \pm SEM was recorded in triplicate on days 0 and 2. (E) The transformed Ba/F3 cells became again dependent on IL-3 for their cell growth after electroporation with an effective siRNA targeting PDGFR $\alpha$. As control, all cell lines were electroporated with a scrambled siRNA. Mean growth \pm SEM was recorded in triplicate on days 0 and 2. Data of the scrambled siRNA are set to $100 \%$; data of the tested siRNAs are relative to the scrambled siRNA.

the Pten shRNAs in parental $\mathrm{Ba} / \mathrm{F} 3$ cells and $\mathrm{Ba} / \mathrm{F} 3$ cells expressing p16-tPDGFR $\alpha$. The different Pten shRNAs had no effect on the control cells nor on the cells containing the p16-tPDGFR $\alpha$ fusion, confirming that the observed effects were specific (Fig. 3A).

Toxic off-target effects of a shRNA can be identified by simply adding IL-3 to the transformed cells, which makes the cells independent from the $\operatorname{tPDGFR} \alpha$ protein by stimulating their proliferation and survival through the IL-3 receptor. Specific shRNAs that target p16 or Pten in the tPDGFR $\alpha$ transcripts should result in inhibition of cell proliferation, and this negative effect on proliferation should be rescued by the addition of IL-3. Indeed, we showed that the cell lines, expressing the p16-tPDGFR $\alpha$ or Pten-tPDGFR $\alpha$ fusion proteins, could proliferate in the presence of IL-3 after transduction with the effective shRNA-p16-1 or shRNA-Pten-4, but not in the absence of IL-3 (Fig. 3B). These data confirm that the effect that we observe of the shRNAs in the absence of IL-3 are not due to a nonspecific effect of the shRNAs.

The data of the proliferation assay were confirmed by protein expression analysis in transformed $\mathrm{Ba} / \mathrm{F} 3$ cells containing p16-tPDGFR $\alpha$ transduced with the shRNAs targeting p16, and, similarly, in cells containing Pten-tPDGFR $\alpha$ transduced with shRNAs targeting Pten. As expected, the effective shRNA-p16-1 and shRNA-p16-3 resulted in a significant decrease of the protein expression of p16-tPDGFR $\alpha$, while there was no down-regulation of p16-tPDGFR $\alpha$ protein levels by shRNA-p16-2 (Fig. 3C). Furthermore, the strongest down-regulation of the Pten-tPDGFR $\alpha$ fusion gene was observed for shRNA-Pten-4. To a lesser extent, shRNA-Pten-2 and shRNA-Pten-3 also resulted in decreased protein expression of Pten-tPDGFR $\alpha$. There was no knockdown in the control experiments where $\mathrm{Ba} / \mathrm{F} 3$ cells with p16tPDGFR $\alpha$ were transduced with Pten shRNAs or where $\mathrm{Ba} / \mathrm{F} 3$ 
A

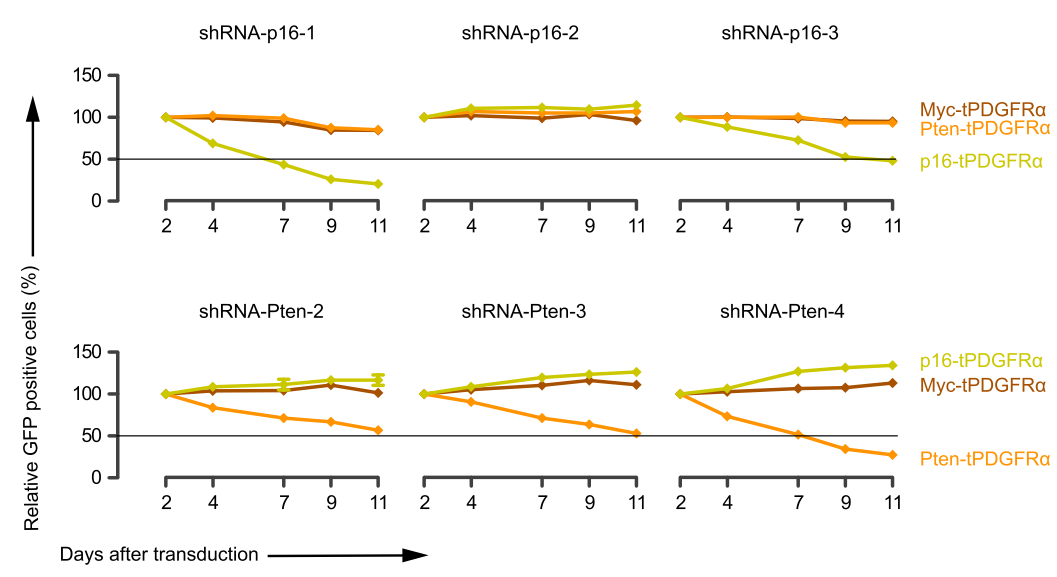

B

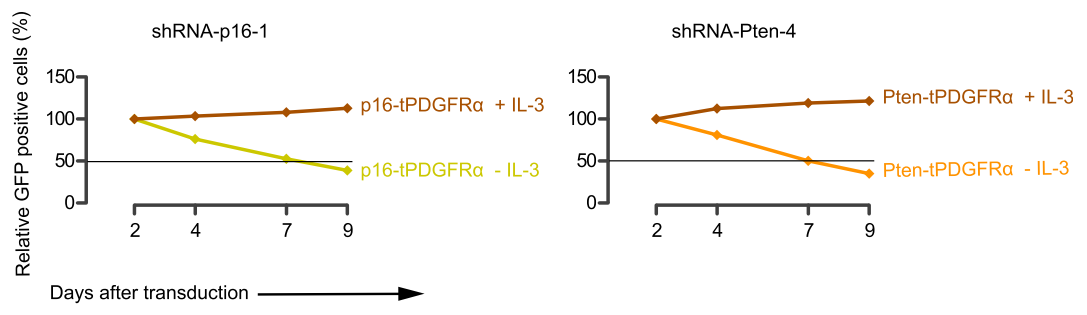

C

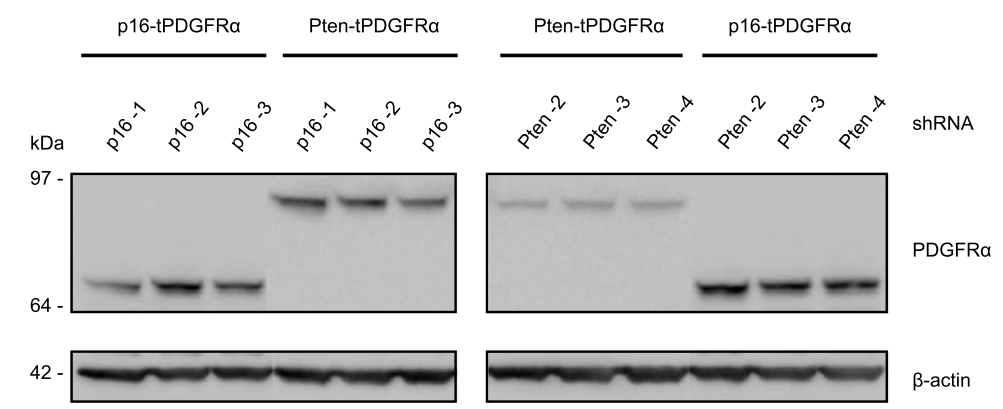

FIGURE 3. Screening of shRNAs using Pten-tPDGFR $\alpha$ and p16-tPDGFR $\alpha$ transformed Ba/F3 cells. (A) Effective shRNAs lead to a decrease in the relative amount of GFP positive cells in contrast to noneffective shRNAs. Mean relative amount of GFP positive cells \pm SEM was recorded in triplicate on days 2, 4, 7, 9, and 11 . All values on day 2 are set to $100 \%$, all other values are relative to day 2. (B) The proliferation of Ba/F3 cells expressing the p16-tPDGFR $\alpha$ or Pten-tPDGFR $\alpha$ fusion proteins was inhibited after expression of shRNA-p16-1 or shRNA-Pten-4, and this inhibition could be rescued by the addition of IL-3. Mean relative amount of GFP positive cells \pm SEM was recorded in triplicate on days $2,4,7,9$, and 11 . All values on day 2 are set to $100 \%$, all other values are relative to day 2. (C) Down-regulation of Pten-tPDGFR $\alpha$ and p16-tPDGFR $\alpha$ protein expression in transformed $\mathrm{Ba} / \mathrm{F} 3$ cells transduced with shRNAs against p16 and Pten. show that the position of the target gene indeed matters. Although shRNA-p16-1 could potently decrease the proliferation of $\mathrm{Ba} / \mathrm{F} 3$ cells expressing p16-tPDGFR $\alpha$, much less effect was observed with the shRNA in cells expressing tPDGFR $\alpha$ p16 or tPDGFR $\alpha^{*}$-p16 (Fig. 4A). Similarly, shRNA-Pten-4 resulted in a substantially lower decrease in the amount of GFP positive cells in experiments with Pten-tPDGFR $\alpha$ compared with tPDGFR $\alpha$ Pten or tPDGFR $\alpha^{\star}$-Pten (Fig. 4A). The same results were observed on a protein level when the genes of interest were placed in front of $t P D G F R \alpha$. After transduction with shRNA-p16-1, reduction in p16-tPDGFR $\alpha$ expression level was more pronounced compared with tPDGFR $\alpha$ p16 or tPDGFR $\alpha^{\star}-$ p16. No knockdown of the control constructs containing Pten was observed with shRNAs targeting $p 16$. Also, the protein level of Pten-tPDGFR $\alpha$ was more reduced after shRNA-Pten-4 transduction compared with $\operatorname{tPDGFR} \alpha-$ Pten or tPDGFR $\alpha^{\star}$-Pten. There was no knockdown of the control constructs containing $p 16$ with shRNAs targeting Pten (Fig. 4B). Similar results were obtained for the other shRNAs (data not shown). These data demonstrate that for the proliferation read-out, the constructs in which the gene of interest are positioned at the $5^{\prime}$ end of the mRNA, upstream of $t P D G F R \alpha$, are to be preferred. These results also confirm that we do not obtain similar knockdown effects for the three different variants of the system and, thus, that knockdown efficiency is dependent on the position of the target sequence in the mRNA.

\section{Selection of siRNAs targeting p16 and Pten using the $\mathrm{Ba} / \mathrm{F} 3$ validation system}

cells with Pten-tPDGFR $\alpha$ were transduced with p16 shRNAs, indicating the specificity of the system (Fig. 3C).

For the experiments described above, we only used the constructs in which the gene of interest (p16 or Pten) was cloned upstream of $t P D G F R \alpha$, generating p16-tPDGFR $\alpha$ or Pten-tPDGFR $\alpha$ fusion proteins. Next, we determined whether the system was also functioning in cases where the gene of interest was cloned downstream from $t P D G F R \alpha$, either as fusion protein or downstream from the stop codon of tPDGFR $\alpha$ (thus, in the $3^{\prime}$ UTR of tPDGFR $\alpha$ ). Our results
In addition to testing of shRNAs, we also applied our validation system for the selection of effective siRNAs by electroporation of the $\mathrm{Ba} / \mathrm{F} 3$ cells (Fig. 1B' $\mathrm{B}^{\prime}$. We electroporated the $\mathrm{Ba} / \mathrm{F} 3$ cells expressing p16-tPDGFR $\alpha$ or PtentPDGFR $\alpha$ with 10 siRNAs directed against $p 16$ and 10 siRNAs directed against Pten, respectively. Two days after electroporation, we measured the growth of the cells, which revealed major differences between the different siRNAs (Fig. 5A). Four of these siRNAs were tested in more detail (Fig. 5A, indicated in orange) and effects were confirmed 
A
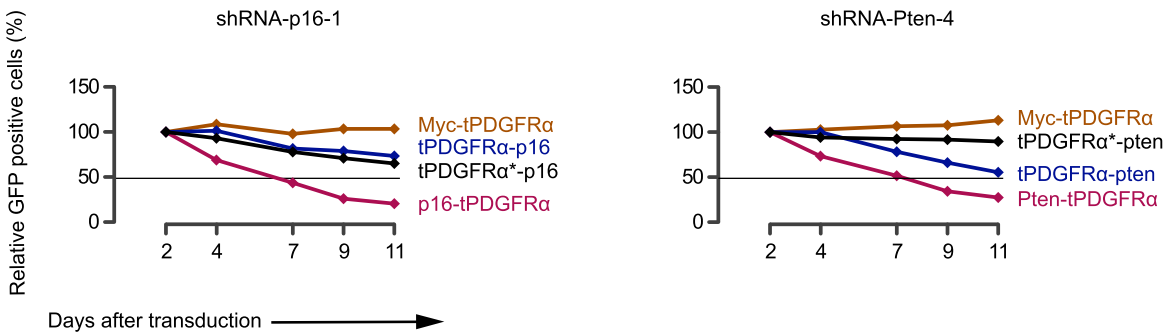

B
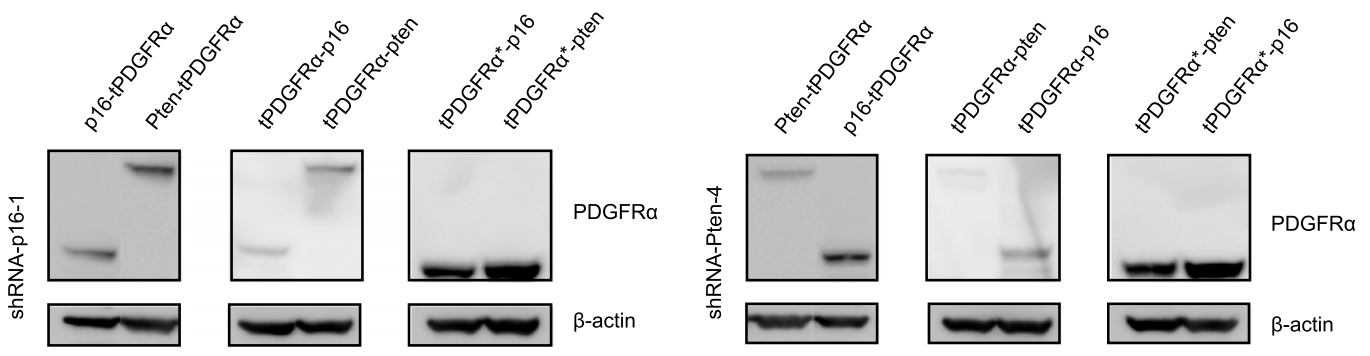

FIGURE 4. Screening of shRNAs directed against Pten and $p 16$ results in different outcomes according to the position of the gene of interest in the tPDGFR $\alpha$ constructs. (A) Effective shRNAs result in a decrease of the relative amount of GFP positive cells when Pten and p16 are placed upstream of the tPDGFR $\alpha$. A less clear effect is observed when Pten and $p 16$ are downstream from the tPDGFR $\alpha$. Mean relative amount of GFP positive cells \pm SEM was recorded in triplicate on days $2,4,7,9$, and 11 . All data on day 2 are set to $100 \%$, and all other data are relative to day 2 . (B) Downregulation of tPDGFR $\alpha$ and tPDGFR $\alpha$ fusion proteins in the transformed Ba/F3 cells transduced with shRNA-p16-1 or shRNA-Pten-4.

in a second experiment (Fig. 5B). The growth reduction was the highest for siRNA-p16-3 and siRNA-Pten-9, respectively. SiRNA-p16-4, siRNA-p16-5, siRNA-Pten-1, and siRNAPten-2 were less effective in reducing the growth of the cells. The noneffective siRNA-p16-D and siRNA-Pten-A were not able to reduce the growth in the transformed $\mathrm{Ba} / \mathrm{F} 3$ cells containing the p16-tPDGFR $\alpha$ or Pten-tPDGFR $\alpha$, respectively (Fig. 5B). As also observed for the shRNAs, the effect of the siRNAs on cell proliferation was less pronounced when p16 and Pten were placed downstream from the tPDGFR $\alpha$ (data not shown).

As shown for the shRNA experiments, cell lines electroporated with siRNAs can be grown in the presence of IL-3 to distinguish between specific and toxic effects. The cell lines expressing p16-tPDGFR $\alpha$ or Pten-tPDGFR $\alpha$ survived after electroporation with an effective siRNA against $p 16$ or Pten when IL-3 was added (Fig. 5C). These data confirm that the siRNAs produce the inhibitory effect on proliferation of the cells specifically through the knockdown of the p16-tPDGFR $\alpha$ or Pten-tPDGFR $\alpha$ transcripts, and not due to a specific knockdown of critical genes needed for proliferation or survival.

In general, the knockdown effect on protein expression (Fig. 5D) correlated well with the effect on cell proliferation (Fig. 5B). Indeed, clear down-regulation of expression of the p16-tPDGFR $\alpha$ or Pten-tPDGFR $\alpha$ fusion proteins was observed when the cell lines were electroporated with the siRNAs that had a clear effect on the proliferation of the cells, while less or no knockdown was observed for siRNAs not having much effect on the proliferation. (Fig. 5B,D). These data confirm the correlation between effects on protein expression and effects on cell proliferation in the $\mathrm{Ba} / \mathrm{F} 3$ system.

\section{Correlation of the knockdown efficiency in the Ba/F3 cell system with knockdown of endogenous Pten or p16 expression in mouse cell lines}

Previous experiments demonstrated the potential use of the $\mathrm{Ba} / \mathrm{F} 3$ system to screen for functional siRNAs or shRNAs, but did not address the correlation between the results in the $\mathrm{Ba} / \mathrm{F} 3$ cell system and the effects on the normal endogenous transcripts of Pten or Cdkn2a. To investigate whether the results of the $\mathrm{Ba} / \mathrm{F} 3$ system reflect the knockdown efficiencies obtained on endogenous transcripts, we next performed experiments with parental $\mathrm{Ba} / \mathrm{F} 3$ cells and MOHITO cells (Kleppe et al. 2011). We determined whether the effective shRNAs and siRNAs, validated in the system, were also able to down-regulate endogenous Pten transcripts in $\mathrm{Ba} / \mathrm{F} 3$ cells. Due to the absence of endogenous p16 expression in $\mathrm{Ba} / \mathrm{F} 3$ cells, we used endogenous $C d k n 2 a$ ( $p 16)$ transcripts in MOHITO cells to validate the p16 RNAi molecules.

Parental $\mathrm{Ba} / \mathrm{F} 3$ cells were transduced with the shRNAs targeting Pten, and knockdown of Pten transcripts was measured by detection of Pten protein expression. The three shRNAs produced strong knockdown of Pten (Fig. $6 \mathrm{~A}$, left), which correlated well with effects observed in the $\mathrm{Ba} / \mathrm{F} 3$ validation system (Fig. 3), where all three shRNAs 
A
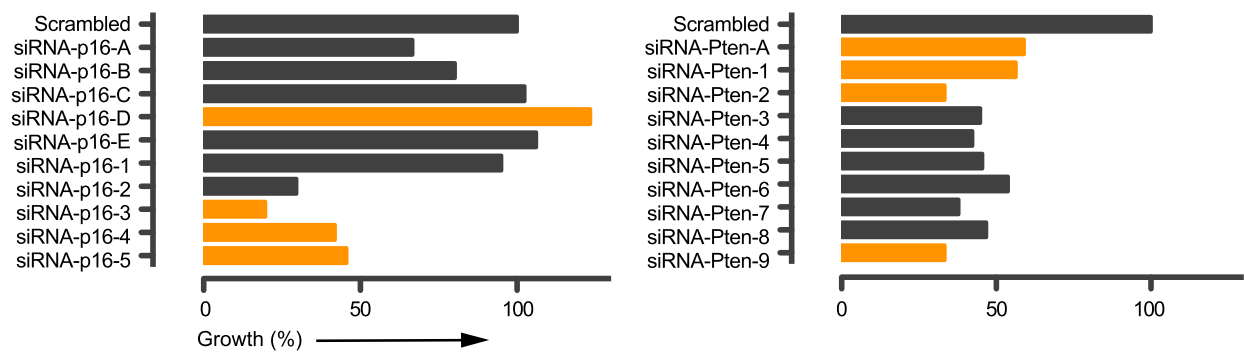

B

C
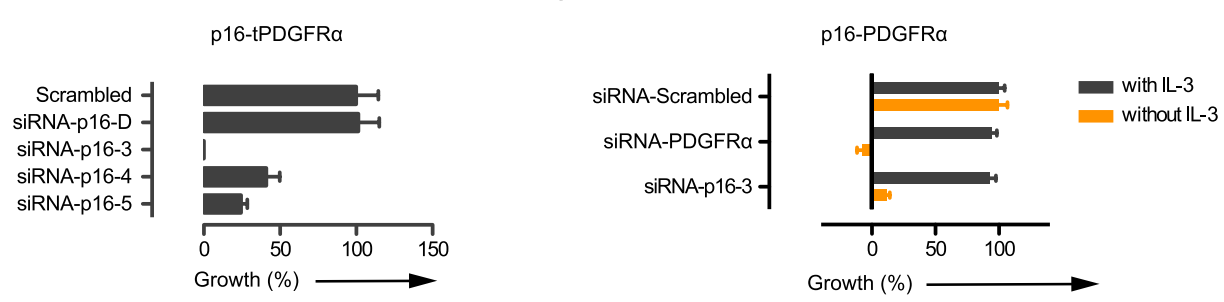

Pten-tPDGFR $\alpha$

Pten-PDGFRa

Scrambled -

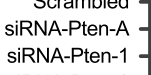
siRNA-Pten-2 siRNA-Pten-9 -
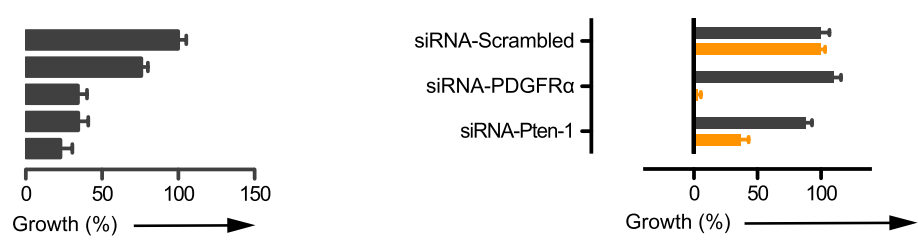

D
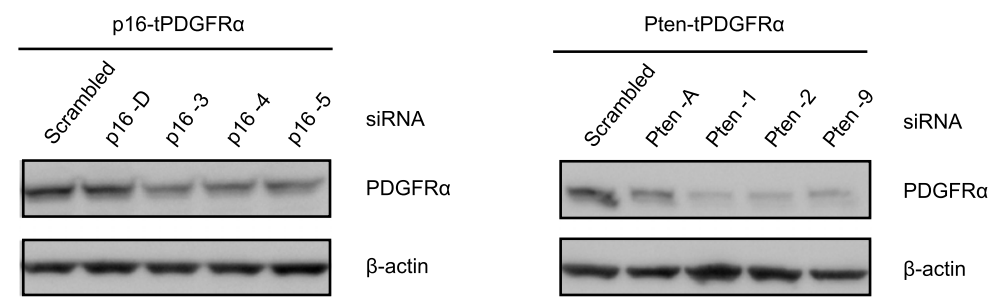

FIGURE 5. Screening and validation of siRNAs. (A) Ten siRNAs directed against Pten and $p 16$ were screened in the p16-tPDGFR $\alpha$ and the PtentPDGFR $\alpha$ transformed $\mathrm{Ba} / \mathrm{F} 3$ cells, respectively. The siRNAs in orange were confirmed in a second experiment $(B)$. (B) Effective siRNAs result in reduced growth of p16-tPDGFR $\alpha$ and Pten-tPDGFR $\alpha$ transduced Ba/F3 cells. Mean growth \pm SEM was recorded fivefold on days 0 and 2. Data of the scrambled siRNA are set to $100 \%$, data of the tested siRNAs are relative to the scrambled siRNA. $(C)$ The proliferation of $\mathrm{Ba} / \mathrm{F} 3$ cells expressing the fusion protein p16-tPDGFR $\alpha$ or Pten-tPDGFR $\alpha$ was inhibited after electroporation with the effective siRNA-p16-3 or siRNAPten-1, respectively, and this inhibition could be rescued by the addition of IL-3. As control, all cell lines were electroporated with a scrambled siRNA. Mean growth \pm SEM was recorded in triplicate on days 0 and 2 . Data of the scrambled siRNA are set to $100 \%$, and the data of the tested siRNAs are relative to the scrambled siRNA. (D) Effective siRNA gave a reduction in the protein expression of p16-tPDGFR $\alpha$ and Pten-tPDGFR $\alpha$.

also showed strong effects, with the shRNA4 being the most potent.

Decrease in the endogenous Pten expression was also observed when $\mathrm{Ba} / \mathrm{F} 3$ cells were electroporated with the siRNAs targeting Pten. Compared with the scrambled siRNA, the decrease in expression was minimal when electroporated with the siRNA-Pten-A, and a strong knockdown was observed with siRNA-Pten-1, siRNA-Pten-2, and siRNA-Pten-9 (Fig. $6 \mathrm{~A}$, right). These data recapitulated well the data observed with the Pten-tPDGFR $\alpha$ expressing Ba/F3 cells (Fig. 5).

Similarly, also for the p16 shRNAs, a good correlation was observed between the knockdown of the p16 transcript in
MOHITO cells (Fig. 6B, left) and the $\mathrm{Ba} / \mathrm{F} 3$ validation system experiments (Fig. 3), in which shRNA-p16-1 was indicated as the most efficient shRNA.

Finally, knockdown efficiencies of the p16 siRNAs were tested on endogenous p16 expression in the MOHITO cell line. We observed the best knockdown for siRNA-p16-3 (Fig. $6 \mathrm{~B}$, right), as also observed in the $\mathrm{Ba} / \mathrm{F} 3$ validation system (Fig. 5B), while the knockdowns for the other siRNAs were slightly different from those observed in the validation system (Fig. 6B, right). Thus, overall, the knockdown effects observed for the endogenous transcripts correlated very well to the knockdown measured with the $\mathrm{Ba} / \mathrm{F} 3$ validation system. 
A

$\mathrm{Ba} / \mathrm{F3}$ cells
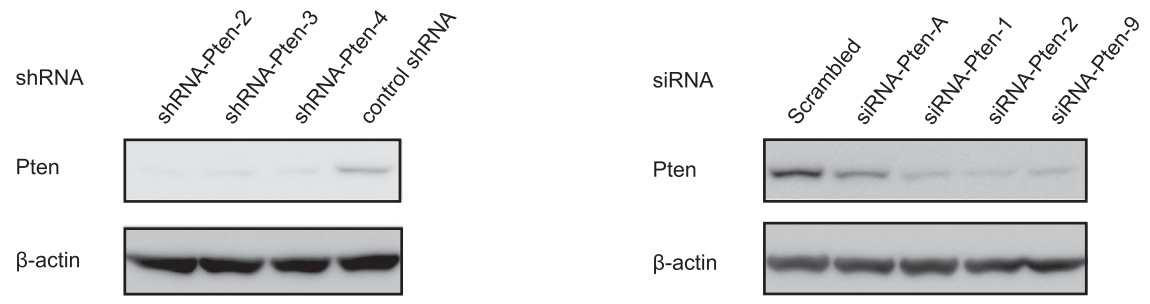

$\mathbf{B}$
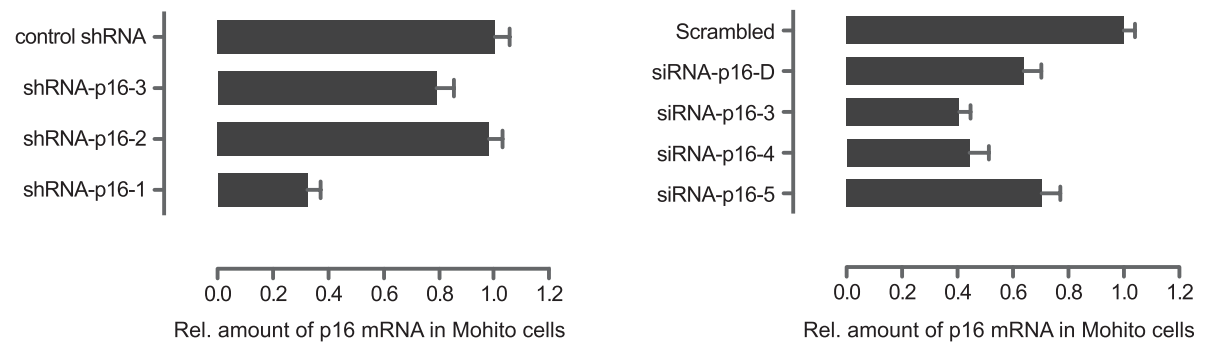

FIGURE 6. Down-regulation of endogenous Pten and p16 in Ba/F3 cells and in MOHITO cells. (A) Down-regulation of endogenous Pten expression in $\mathrm{Ba} / \mathrm{F} 3$ cells by shRNAs (left) and by siRNAs (right). The control shRNA mentioned at left is shRNA-p16-1. (B) Reduction in endogenous p16 transcripts in MOHITO cells by shRNAs (left) and by siRNAs (right). The control shRNA at left is shRNA-Pten-4.

\section{DISCUSSION}

Since the discovery of RNAi, gene silencing has been used with increasing interest in both research and therapeutic applications. Over the last years, many algorithms for shRNA and siRNA design have been developed, all based on empirical rules. While these algorithms assist in the selection of RNAi molecules, none of the currently available algorithms can predict the efficiency of siRNAs and shRNAs with high accuracy (Pei and Tuschl 2006; Krueger et al. 2007). Here, we describe the development and evaluation of a cell-based validation system to complement classical design algorithms to make better choices for effective gene silencing by means of RNAi.

The system we describe here is based on the transforming properties of a truncated form of the PDGFR $\alpha$ kinase (tPDGFR $\alpha$ ) in $\mathrm{Ba} / \mathrm{F} 3$ cells. Ba/F3 cells expressing tPDGFR $\alpha$ proliferate fast (doubling time $<24 \mathrm{~h}$ ) and are completely dependent on the presence of tPDGFR $\alpha$ for their proliferation and survival. We hypothesized that by putting the gene of interest on the same transcript as tPDGFR $\alpha$, we could generate a sensor for efficiency of knockdown of the gene of interest. Effective shRNAs and siRNAs, introduced by transduction or electroporation, respectively, target the gene of interest that results in down-regulation of the tPDGFR $\alpha$ fusion protein. Loss of tPDGFR $\alpha$ will block proliferation and survival of the $\mathrm{Ba} / \mathrm{F} 3$ cells, which is an easy readout to measure in these rapidly proliferating cells.
Our results show that this is indeed possible. We tested the system by cloning the coding sequences of mouse Cdkn2a (p16) or mouse Pten on the tPDGFR $\alpha$ transcript (Fig. 2). Combining two RNA fragments in a transcript may alter the RNA secondary structure, which can affect binding of the RNAi molecules to the target RNA sequence. For this reason we tested three different variants for each gene of interest (Wang et al. 2009). We generated (1) p16-tPDGFR $\alpha$ or Pten-tPDGFR $\alpha$ fusion transcripts that encode p16tPDGFR $\alpha$ or Pten-tPDGFR $\alpha$ fusion proteins, (2) tPDGFR $\alpha$ p16 or tPDGFR $\alpha$-Pten fusion transcripts that encode tPDGFR $\alpha$-p16 or tPDGFR $\alpha$-Pten fusion proteins, or (3) tPDGFR $\alpha^{*}$-p16 or $t P D G F R \alpha^{*}$-Pten fusion transcripts that will express only the tPDGFR $\alpha$ protein, since a stop codon is present at the end of the coding sequence of tPDGFR $\alpha$ and the p16 or Pten sequences are thus present in the $3^{\prime}$ UTR of this transcript. The documented knockdown effects on protein level and on proliferation of the cells were much more pronounced in the first variant with the p16-tPDGFR $\alpha$ and Pten-tPDGFR $\alpha$ fusion proteins (Fig. 4). We therefore recommend using this setup for future experiments.

We realize that our screening method is an artificial system and therefore has a few disadvantages. It is possible that RNAi molecules with a good knockdown efficiency in the system will not reach this level of knockdown when targeting the endogenous transcripts. Nevertheless, in this study we observed a good correlation between the knockdown efficiency of p16 and Pten obtained in the screening system and the knockdown of endogenous p16 and Pten in mouse cell lines. 
In this study we showed that our validation system is a reliable system that is able to identify efficient shRNAs and siRNAs. From three tested shRNAs directed against p16, only one shRNA was identified as a shRNA with strong RNAi effect, and this shRNA was confirmed to function well to down-regulate the expression of the endogenous p16 transcript in MOHITO cells. Similarly, a good correlation between the results in the $\mathrm{Ba} / \mathrm{F} 3$ validation system and knockdown effects on the endogenous gene was observed for Pten as well. The validation system detected clear differences between different siRNAs or shRNAs, and a strong correlation was documented with effects on the endogenous transcripts. These data support the $\mathrm{Ba} / \mathrm{F} 3$ validation system as a potent and easy-to-use system to select siRNAs or shRNAs with a good knockdown effect.

Our system has a few important differences compared with experimental validation systems described in the past. In those systems, a reporter assay is used where a target gene is fused to a fluorescent or enzymatic reporter gene, like EGFP or luciferase, respectively. Reduction in the fluorescent or enzymatic activity is observed when effective shRNAs or siRNAs target the gene of interest (Kumar et al. 2003; Huesken et al. 2005; Zhao et al. 2005; Hung et al. 2006; Kasim et al. 2006; Malik et al. 2006; Luo et al. 2007; Fellmann et al. 2011). The read-out of our system is the reduction in cell growth due to down-regulation of the tPDGFR $\alpha$ protein kinase fused to the target gene. Looking at the direct cellular effects might have some advantages, since protein levels in artificial systems may not always correlate with the cellular effects.

A major challenge for many validation systems is the low transfection or transduction efficiencies, which make it difficult to interpret and compare results of the different shRNAs and siRNAs. A solution to overcome this problem is to include a reference gene (Huesken et al. 2005; Zhao et al. 2005; Hung et al. 2006; Kasim et al. 2006; Malik et al. 2006), but this makes the cloning experiments and the interpretation of results more complex. In our system, siRNA and shRNA delivery is not a major problem: Transfection of siRNAs occurs through electroporation, which is highly efficient in the $\mathrm{Ba} / \mathrm{F} 3$ suspension cell line. It was also not necessary to correct for differences in transduction efficiency, since we could perfectly measure the transduced cells only. All shRNA vectors also contained the GFP gene, so that we could gate on GFP positive cells for the shRNA experiments. So, even with low transduction efficiency, we were able to observe differences between the different shRNAs. In addition, another advantage of using GFP labeled cells, instead of measuring luciferase activity, is the possibility of measuring several consecutive time points (over several days) with the same set of cells and the direct detection of fluorescence (Malik et al. 2006).

A second advantage of the $\mathrm{Ba} / \mathrm{F} 3$ cell system is that the cells can be made independent from the transcripts of interest (here the $t P D G F R \alpha$ fusion transcript) by the addition of IL-3. IL-3 is the ligand for the IL-3 receptor, which is expressed by the $\mathrm{Ba} / \mathrm{F} 3$ cells and which can be used to drive the proliferation and the survival of the cells. In this way, addition of IL-3 will make the cells independent from the $t P D G F R \alpha$ fusion transcript, which can be used to investigate whether the inhibitory effect observed by the RNAi molecules is due to a specific effect on the tPDGFR $\alpha$ fusion transcript or rather due to a nonspecific effect. It is plausible that some shRNAs or siRNAs could target critical transcripts in the cell and in this way interfere with the proliferation of the $\mathrm{Ba} / \mathrm{F} 3$ cells. This would initially cause a false-positive readout in this system. However, when experiments are performed in the absence and also in the presence of IL-3, these false-positive results can be detected easily. Finally, it could also be that the shRNA or siRNA target the PDGFR $\alpha$ part of the transcript, rather than the part of the gene of interest. Again, this can be easily detected by using the cells expressing the myc-tPDGFR $\alpha$ fusion, as these cells should not be inhibited by shRNAs or siRNAs specifically targeting the gene of interest.

We can conclude that our validation system is an easy and reliable system to distinguish effective and noneffective shRNAs and siRNAs, and that this system can be used for the selection of the siRNAs and shRNAs with the appropriate knockdown characteristics.

\section{MATERIALS AND METHODS}

\section{Constructs}

A myc-tagged truncated version of PDGFR $\alpha$ (tPDGFR $\alpha$ ) was cloned in the MSCV-puro vector (Clontech). The coding sequences of mouse Pten and Cdkn2a (p16) were amplified by PCR and cloned into the MSCV-puro vector already containing tPDGFR $\alpha$ (Fig. 2A). The CmiR3 vector was generated by cloning the mir30 backbone in the MSCV-GFP vector.

\section{shRNA and siRNA}

The shRNA-Pten-4 was purchased from Open Biosystems. All of the other shRNAs targeting Pten and p16 were designed using the online available RNAi Central (http://katahdin.cshl.org/ homepage/siRNA/RNAi.cgi?type=shRNA), and the oligonucleotide sequences were purchased from Integrated DNA Technologies (IDT). All shRNAs were cloned into the CmiR3 vector. The amount of GFP positive cells was measured by flow cytometry. GFP positive cells were sorted with the FACSsorter to investigate the effect of the shRNAs on the endogenous protein expression. The sequences of the shRNAs and siRNAs are listed in Supplemental Tables 1 and 2 .

\section{Cell lines, virus production, and retroviral transduction}

HEK293T cells were grown in Dulbecco's modified Eagle's medium with $10 \%$ fetal calf serum. Virus production and retroviral trans- 
duction were performed as described previously (De Keersmaecker et al. 2005). The transformed $\mathrm{Ba} / \mathrm{F} 3$ cells were cultured in RPMI1640 with $10 \%$ fetal calf serum. Untransformed $\mathrm{Ba} / \mathrm{F} 3$ cells were cultured in RPMI-1640 with 10\% fetal calf serum and $1 \mathrm{ng} / \mathrm{mL}$ of IL-3 (Peprotech). For analysis of PDGFR $\alpha$ dependency, transformed $\mathrm{Ba} / \mathrm{F} 3$ cells were treated with $10 \mathrm{nM}$ imatinib (ChemieTek).

\section{Western blotting}

The cells were lysed in cold lysis buffer containing $5 \mathrm{mM} \mathrm{NA}_{3} \mathrm{VO}_{4}$ and protease inhibitors (Complete tablets, Roche). The proteins were separated on NuPAGE NOVEX Bis-Tris 4\%-12\% gels (Invitrogen) and transferred to PVDF membranes. Subsequent Western blot analysis was done using primary antibodies directed against PDGFR $\alpha$, phospho-PDGFR $\alpha$, and $\beta$-actin (Santa Cruz).

\section{Electroporation and siRNA knockdown}

For electroporation, $1.5 \times 10^{6} \mathrm{Ba} / \mathrm{F} 3$ cells were resuspended in $400 \mu \mathrm{L}$ of serum-free medium and transferred to 4 -mm cuvettes (Biorad). The electroporation parameters were as follows: exponential decay, $950 \mu \mathrm{F}$ and $300 \mathrm{~V}$. The electroporated cells were transferred to 12 -well plates containing $2 \mathrm{~mL}$ of pre-warmed RPMI supplemented with $10 \%$ FCS. Growth reduction was analyzed using the Vi-cell XR cell viability analyzer (Beckman Coulter) $1 \mathrm{~h}$ and 48 $\mathrm{h}$ after electroporation. Predesigned and custom-designed siRNA duplexes were purchased from IDT.

\section{Quantitative PCR}

Fast SYBR Green Master Mix (Applied Biosystems) was used for performance of quantitative real-time PCR with the LightCycler 480 Real-Time PCR System (Roche Diagnostics). Hprt gene was used as housekeeping gene. Data were analyzed with the LC480 software (Roche Diagnostics).

\section{SUPPLEMENTAL MATERIAL}

Supplemental material is available for this article.

\section{ACKNOWLEDGMENTS}

This work was supported by the Foundation against Cancer (SCIE2006-34) and the European Research Council (ERC-starting grant).

Received August 30, 2011; accepted March 14, 2012.

\section{REFERENCES}

Cools J, DeAngelo DJ, Gotlib J, Stover EH, Legare RD, Cortes J, Kutok J, Clark J, Galinsky I, Griffin JD, et al. 2003a. A tyrosine kinase created by fusion of the PDGFRA and FIP1L1 genes as a therapeutic target of imatinib in idiopathic hypereosinophilic syndrome. $N$ Engl J Med 348: 1201-1214.

Cools J, Stover EH, Boulton CL, Gotlib J, Legare RD, Amaral SM, Curley DP, Duclos N, Rowan R, Kutok JL, et al. 2003b. PKC412 overcomes resistance to imatinib in a murine model of FIP1L1-PDGFR $\alpha$ induced myeloproliferative disease. Cancer Cell 3: 459-469.
De Keersmaecker K, Graux C, Odero MD, Mentens N, Somers R, Maertens J, Wlodarska I, Vandenberghe P, Hagemeijer A, Marynen P, et al. 2005. Fusion of EML1 to ABL1 in T-cell acute lymphoblastic leukemia with cryptic $\mathrm{t}(9 ; 14)(\mathrm{q} 34 ; \mathrm{q} 32)$. Blood 105: 4849-4852.

Elbashir SM, Harborth J, Lendeckel W, Yalcin A, Weber K, Tuschl T. 2001. Duplexes of 21-nucleotide RNAs mediate RNA interference in cultured mammalian cells. Nature 411: 494-498.

Fellmann C, Zuber J, McJunkin K, Chang K, Malone CD, Dickins RA, $\mathrm{Xu}$ Q, Hengartner MO, Elledge SJ, Hannon GJ, et al. 2011. Functional identification of optimized RNAi triggers using a massively parallel Sensor assay. Mol Cell 41: 733-746.

Holen T, Amarzguioui M, Wiiger MT, Babaie E, Prydz H. 2002. Positional effects of short interfering RNAs targeting the human coagulation trigger Tissue Factor. Nucleic Acids Res 30: 17571766.

Huesken D, Lange J, Mickanin C, Weiler J, Asselbergs F, Warner J, Meloon B, Engel S, Rosenberg A, Cohen D, et al. 2005. Design of a genome-wide siRNA library using an artificial neural network. Nat Biotechnol 23: 995-1001.

Hung C-F, Lu K-C, Cheng T-L, Wu R-H, Huang L-Y, Teng C-F, Chang W-T. 2006. A novel siRNA validation system for functional screening and identification of effective RNAi probes in mammalian cells. Biochem Biophys Res Commun 346: 707-720.

Kapadia SB, Brideau-Andersen A, Chisari FV. 2003. Interference of hepatitis $\mathrm{C}$ virus RNA replication by short interfering RNAs. Proc Natl Acad Sci 100: 2014-2018.

Kasim V, Taira K, Miyagishi M. 2006. Screening of siRNA target sequences by using fragmentized DNA. J Gene Med 8: 782791.

Kleppe M, Mentens N, Tousseyn T, Wlodarska I, Cools J. 2011. MOHITO, a novel mouse cytokine-dependent T-cell line, enables studies of oncogenic signaling in the T-cell context. Haematologica 96: 779-783.

Krol J, Loedige I, Filipowicz W. 2010. The widespread regulation of microRNA biogenesis, function and decay. Nat Rev Genet 11: 597610.

Krueger U, Bergauer T, Kaufmann B, Wolter I, Pilk S, Heider-Fabian M, Kirch S, Artz-Oppitz C, Isselhorst M, Konrad J. 2007. Insights into effective RNAi gained from large-scale siRNA validation screening. Oligonucleotides 17: 237-250.

Kumar R, Conklin DS, Mittal V. 2003. High-throughput selection of effective RNAi probes for gene silencing. Genome Res 13: 23332340.

Lierman E, Lahortiga I, Van Miegroet H, Mentens N, Marynen P, Cools J. 2007. The ability of sorafenib to inhibit oncogenic PDGFR $\beta$ and FLT3 mutants and overcome resistance to other small molecule inhibitors. Haematologica 92: 27-34.

Luo Q, Kang Q, Song W-X, Luu HH, Luo X, An N, Luo J, Deng Z-L, Jiang W, Yin $H$, et al. 2007. Selection and validation of optimal siRNA target sites for RNAi-mediated gene silencing. Gene 395: $160-169$.

Malik I, Garrido M, Bähr M, Kügler S, Michel U. 2006. Comparison of test systems for RNAinterference. Biochem Biophys Res Commun 341: 245-253.

Pecot CV, Calin GA, Coleman RL, Lopez-Berestein G, Sood AK. 2011. RNA interference in the clinic: challenges and future directions. Nat Rev Cancer 11: 59-67.

Pei Y, Tuschl T. 2006. On the art of identifying effective and specific siRNAs. Nat Methods 3: 670-676.

Rao DD, Vorhies JS, Senzer N, Nemunaitis J. 2009. siRNA vs. shRNA: similarities and differences. Adv Drug Deliv Rev 61: 746-759.

Reynolds A, Leake D, Boese Q, Scaringe S, Marshall WS, Khvorova A. 2004. Rational siRNA design for RNA interference. Nat Biotechnol 22: $326-330$.

Sashital DG, Doudna JA. 2010. Structural insights into RNA interference. Curr Opin Struct Biol 20: 90-97.

Siomi H, Siomi MC. 2009. On the road to reading the RNAinterference code. Nature 457: 396-404. 
Stover EH, Chen J, Folens C, Lee BH, Mentens N, Marynen P, Williams IR, Gilliland DG, Cools J. 2006. Activation of FIP1L1PDGFR $\alpha$ requires disruption of the juxtamembrane domain of PDGFR $\alpha$ and is FIP1L1-independent. Proc Natl Acad Sci 103: 8078-8083.

Wang X, Wang X, Varma RK, Beauchamp L, Magdaleno S, Sendera TJ. 2009. Selection of hyperfunctional siRNAs with improved potency and specificity. Nucleic Acids Res 37: e152. doi: 10.1093/ nar/gkp864.
Warmuth M, Kim S, Gu X-J, Xia G, Adrián F. 2007. Ba/F3 cells and their use in kinase drug discovery. Curr Opin Oncol 19: 55-60.

Winter J, Jung S, Keller S, Gregory RI, Diederichs S. 2009. Many roads to maturity: microRNA biogenesis pathways and their regulation. Nat Cell Biol 11: 228-234.

Zhao H-F, L'Abbé D, Jolicoeur N, Wu M, Li Z, Yu Z, Shen S-H. 2005. High-throughput screening of effective siRNAs from RNAi libraries delivered via bacterial invasion. Nat Methods 2: 967-973. 

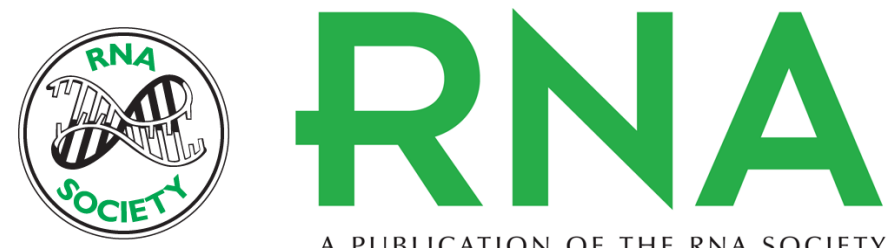

A PUBLICATION OF THE RNA SOCIETY

\section{Development of a siRNA and shRNA screening system based on a kinase fusion protein}

Sofie Pieraets, Luk Cox, Olga Gielen, et al.

RNA 2012 18: 1296-1306 originally published online April 26, 2012

Access the most recent version at doi:10.1261/rna.030015.111

\section{Supplemental http://rnajournal.cshlp.org/content/suppl/2012/03/26/rna.030015.111.DC1 \\ Material}

References This article cites 28 articles, 6 of which can be accessed free at:

http://rnajournal.cshlp.org/content/18/6/1296.full.html\#ref-list-1

\section{License}

Email Alerting Receive free email alerts when new articles cite this article - sign up in the box at the Service top right corner of the article or click here.

\section{III!"II Providing Precise Solutions tor your research.}

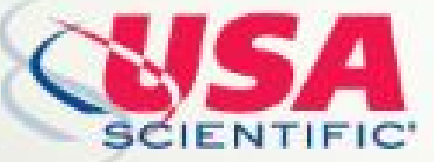

To subscribe to $R N A$ go to:

http://rnajournal.cshlp.org/subscriptions 